\title{
Wiener Filter Used in the EBT2 Film for Radiation Therapy
}

\author{
Liyun Chang
}

\begin{abstract}
EBT2 film has been widely used in the dosimetry application of radiation therapy with lots of benefits especially its self-development, water equivalent, energy independent and high spatial resolution. However, the higher inhomogeneity between the pixels of EBT2 image, needed to be averaged out according to the traditional method, but it could sacrifice the spatial resolution. The Wiener filter technique was introduced in this paper. The EBT2 film was calibrated by using the PDD method and the film calculate doses were compared with the measurement doses by the edge detector with the water phantom. The results demonstrate that the Wiener filter technique has higher accuracy than the traditional method and it would not sacrifice the high spatial resolution of EBT2 film. This method can also be applied to all the quality assurances of treatment planning of radiationtherapy by using EBT2 film.
\end{abstract}

\section{Index Terms-Wiener filter, EBT2 film, radiation therapy}

\section{INTRODUCTION}

A rapid evolution of the available treatment methods has takenplace in radiation therapy during the last two decades, which includes those precise treatment techniques: intensitymodulated(IMRT), stereotactic (SRT) radiotherapy and several dynamic techniques.In order to achieve dose distributions with highlyconformal to the target volume and spare normal tissues (especially the organs at risk), the irregular fieldsand steep dose gradients are applied by the inverse treatment planning system with the advanced linear accelerator. For the effectiveness and success of the treatment prescribed,a high spatial and dosimetric accuracy during treatment delivery is of crucialimportance.Therefore, particular quality assurance (QA) procedureshave been developed together with those increasingly sophisticatedtreatment techniques [1]-[3], which wouldinclude the verification of absolute doses at one or more reference points as well as of twodimensionaldose distributions in different planes in awater-equivalent phantom.

Those requirements could not be achieved by using the conventionaldosimeters, such as ionization chambers, semiconductor detectors and thermoluminescentdetectors (TLDs), since they could not offer high spatial resolution, twodimensionalinformation and determination of absolute dosessimultaneously.Film dosimeters offerpermanent records of the ionizing dose distribution measured at high resolutions with evident advantages for being $2 \mathrm{D}$ detectors. Due to the high-atomic-number material constituents, the

Manuscript received in April 11, 2013; revised June 3, 2013

L. Chang is with the Department of Medical Imaging and Radiological Sciences, I-Shou University, Kaohsiung, Taiwan (e-mail: cliyun@ isu.edu.tw). traditional used silverhalide films, however, have the disadvantage of pronounced energy dependence [4], [5]. This could introduce systematicerrors for most usual treatment plans [6], [7].

With good tissue equivalence, radiochromic filmsuse a radiation-sensitive dye, organized into microcrystalsand embedded in a gelatin binder, to measure the energy dose of ionizing radiations. However, typical fractional doses could not be measured with radio chromic detectors[8].EBT film, the product of International Specialty Products(ISP), which overcomes this limitations, has been widely used specifically to address the needs of the medical physicist and dosimetrist working in the radiotherapy environment[9]-[12].Comparing with previous GAFCHROMIC $^{\circledR}$ films, EBT film is self-developing, but it also incorporates numerous improvements. Some of these improvements include:thedose range extended to be $1 \mathrm{cGy}-800 \mathrm{cGy}$; it is ten times more sensitive than theGAFCHROMICHS film; it is energy independent from the $\mathrm{keV}$ into the $\mathrm{MeV}$ range; thepost-exposure density growth is faster and lower; the temperature withstands up to $70^{\circ} \mathrm{C}$.EBT film can be easily read by standard flatbed scanners andhad a high degree of $2 \mathrm{D}$ spatial accuracy and uniformity of response [13]-[19].

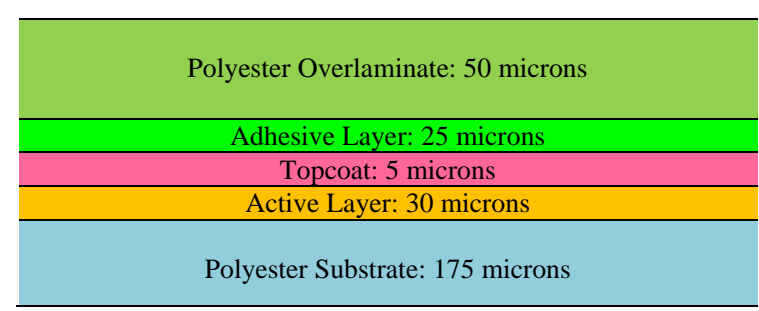

Fig. 1. Configuration of EBT2 film.

EBT2 film is the next generation of EBT film.The configuration of EBT2 film was shown in Fig. 1. The active part of the film has been reduced to a single layer about $30 \mu \mathrm{m}$ and the topcoat is $175 \mu \mathrm{m}$ polyester substrate. EBT2 film uses exactly the same active component as EBT film, but improved at a number of features.

The new film contains a yellow marker dye that protects the active layer from exposure by UV and visible light and reduces the effect from these sources by several times [20] andthe dye could cause minimal intra- and inter-sheet non-uniformity of the films.The coated layers of EBT film, which incorporates a natural gelatin, has been replaced with a synthetic polymer, which reportedly allows better control of the active layer composition, leading to minimal energy dependence[21], [22]. According to manufacturer, the mainly atomic composition of the overall compositionof EBT2 film was listed in Table I and the effective atomic number of overall film, $Z_{e f f}$, is calculated as around 6.8 , which is nearly 
water equivalent and made EBT2 film more suitable for patient dosimetry.

TABLE I: THE MAINLY ATOMIC COMPOSITION OF THE OVERALL COMPOSITIONOF EBT2 FILM (ATOM\%)

\begin{tabular}{cccc}
\hline \hline $\mathrm{H}$ & $\mathrm{Li}$ & $\mathrm{C}$ & $\mathrm{O}$ \\
\hline $40.9 \%$ & $0.1 \%$ & $42.4 \%$ & $16.6 \%$ \\
\hline \hline
\end{tabular}

However, the homogeneity of EBT2 film is still not that good [20], and is getting significant when the distance between the very near two pixels is getting smaller. The general method to reduce the effect of film inhomogeneity is averaging out a lot of pixels; however, it will be a trade-off between the spatial resolution and homogeneity.

Generally speaking, to get better homogeneity, an $8 \times 8$ $\mathrm{mm}^{2}$ area of the film would be taken average for the any centered point[23], but it could sacrifice one of the advantages to utilize EBT2 film for the dosimetry purpose, that is, the high spatial resolution. To keep the high spatial resolution with good film homogeneity, a new algorithm was provided in this paper by filtering the film data with Wiener filter.

\section{MAterials AND MethodS}

Three 8"×10" EBT2 films from Lot No. A03171101B were used for this study. The film was scanned with $127 \mathrm{dpi}$ ( 5 dots per $\mathrm{mm}$ ) before irradiation (pre-scan), which is a good choice for spatial resolution on an Epson 10000XL scanner to generate the background tiff image [24].The $6 \mathrm{MV}$ photon beam of anElecta Synergy clinical linear accelerator was used for this investigation (Fig. 2).

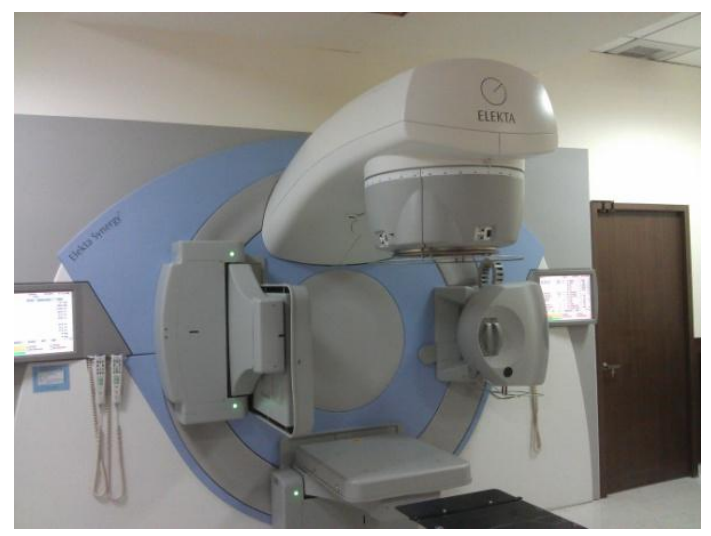

Fig. 2. Electa synergy linear accelerator.

The linear accelerator was calibrated at the depth of $5 \mathrm{~cm}$ withsource-to-surface distance (SSD) $95 \mathrm{~cm}$ for the field size $10 \times 10 \mathrm{~cm}^{2}$ and all related quality assurances were performed according to the AAPM's TG reports[25]-[27] before radiation exposures. But for the setup of EBT2 film, the radiation field size and theSSDwas set as $20 \times 20 \mathrm{~cm}^{2}$ and 100 $\mathrm{cm}$ respectively.

The normalized percentage depth dose (PDD) data with field size $20 \times 20 \mathrm{~cm}^{2}$ was measured by usinga PTW MP3-M water phantom and a Sun Nuclear edge detector, which would be the golden standard for this study, since water phantom is the well-known standard dosimetry calibrationsystem and the edge detector would provide the dosimetry results of extremely high spatial resolution due to its $0.8 \times 0.8 \mathrm{~mm}^{2}$ active detection area.
The film was taped to a $30 \times 30 \times 5 \mathrm{~cm}^{3}$ polystyrene plate and sandwich into the $30 \times 30 \times 30 \mathrm{~cm}^{3}$ polystyrene phantoms as the calibration steps using the PDD method [23]. 290 Monitor Units (MU) of the accelerator were given to each of the films and after that they were scanned again (post-scan) to get the film optical density for the dosimetry analysis, which was performed by Matlab (version 7.12) software.The pixel value was converted from the red channel of the pre-scan and post-scan films to net optical density, OD, which could be written as

$$
O D=\log _{1 D}\left(\frac{P V_{a}}{P V_{b}}\right)
$$

where $P V_{a}$ and $P V_{b}$ were the pixel value of the pre-scan film and the post-scan film, respectively.

The $O D$ on the beam central axis at thedepth 0.4 to $25.0 \mathrm{~cm}$, with an interval of $0.2 \mathrm{~cm}$, was chosen. In all, $124 O D$ points for each film were automatically extracted.The delivered dose versus the measured $O D$ was then could be fitted by thegeneral equation:

$$
D_{\text {fitted dose }}=a 1^{\prime} \times O D+a 2^{\prime} \times O D^{a 3^{\prime}}
$$

where $D_{\text {fitted dose }}$ is the fitted dose; $a 1^{\prime}, a 2^{\prime}$ ' and $a 3^{\prime}$ 'are fitting parameters. To make the fitting process smoothly, the net optical density and the delivered dose were normalized at depth $5 \mathrm{~cm}$, and noted as $N O D$ and $N D$ respectively. The fitting process will be then changed to be

$$
N D=a 1 \times N O D+a 2 \times N O D^{a 3}
$$

where $a 1, a 2$ and $a 3$ are fitting parameters. The fitting process was repeated twice, the first time with $a 1, a 2$ and $a 3$ not bound, but after getting the fitted $a 3$ value, it was rounded to the nearest tenth for the second fitting process.Comparing (2) and (3), the relationship between the fitting parameter in (2) and (3), could be deduced to be that $a 3^{\prime}=a 3, a 1^{\prime}=a 1 \times$ $($ dose at $5 \mathrm{~cm}) /\left(\right.$ net optical density at $5 \mathrm{~cm}$ )and $a 2^{\prime}=a 2 \times$ $($ dose at $5 \mathrm{~cm}) /(\text { net optical density at } 5 \mathrm{~cm})^{a 3}$.

The Wiener filtering process used the function "wiener2 ( $N D,\left[\begin{array}{ll}M & N\end{array}\right]$, noise)" in Matlab. $N$-by- $M$ was the chose neighborhood in the image of matrix $N D$. The Wiener filtered data will be compared with the original data (the data without filtering) and the data averaged with $8 \times 8 \mathrm{~mm}^{2}$.

\section{RESULTS AND DisCUSSIONS}

According empirical experiences, the best choice of $M, N$ and noise would be 30, 30 and 1 respectively, but the filtered matrix should be times 30 to make it back to its original size. The fitting parameters for the three tested EBT2 film was shown in Table II. It is interested that the exponent item in (3) has different value of the third digit after the decimal point. It shows the inter-film discrepanciesin the calibration equation.

The calibration results of the first film by (3) were shown in Fig. 3. The blue dashed curve is thenormalized doseaccording to (3), transferred from thenet optical densitywith $127 \mathrm{dpi}$, which is measured by edge detector in water phantom. That curve is similar to the noises bouncing around the curve of normalized delivered doses, which is the delivered dose normalized at depth $5 \mathrm{~cm}$. Except at the depths of very beginning and very end (usually those are useless data 
at the margins of the film), the curve of Wiener filtered and the $8 \times 8 \mathrm{~mm}^{2}$ averaged are fitted well with the normalized delivered dose. Through Fig. 4, the percentage differences between the normalized delivered doses and the calculated doses for them were also closed each other and within $1.5 \%$

TABLE II: THE FITTING PARAMETERS FOR THE THREE EBT2 FILMS BY (3)

\begin{tabular}{cccc}
\hline \hline & $\mathrm{a} 1$ & $\mathrm{a} 2$ & $\mathrm{a} 3$ \\
\hline Film 1 & 0.5658 & 0.4322 & 1.8 \\
Film 2 & 0.6855 & 0.3193 & 2.0 \\
Film 3 & 0.5347 & $0 . .4776$ & 1.7 \\
\hline \hline
\end{tabular}

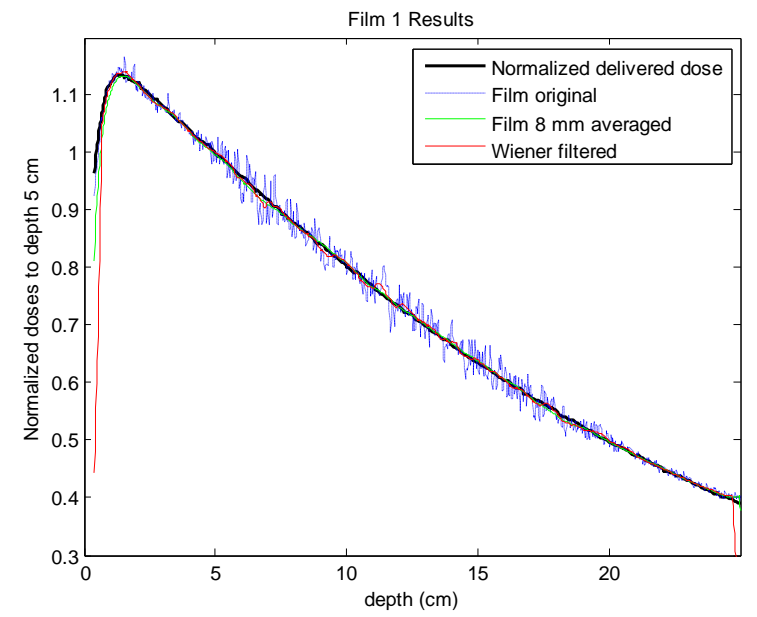

Fig. 3. The calibration results of the first film

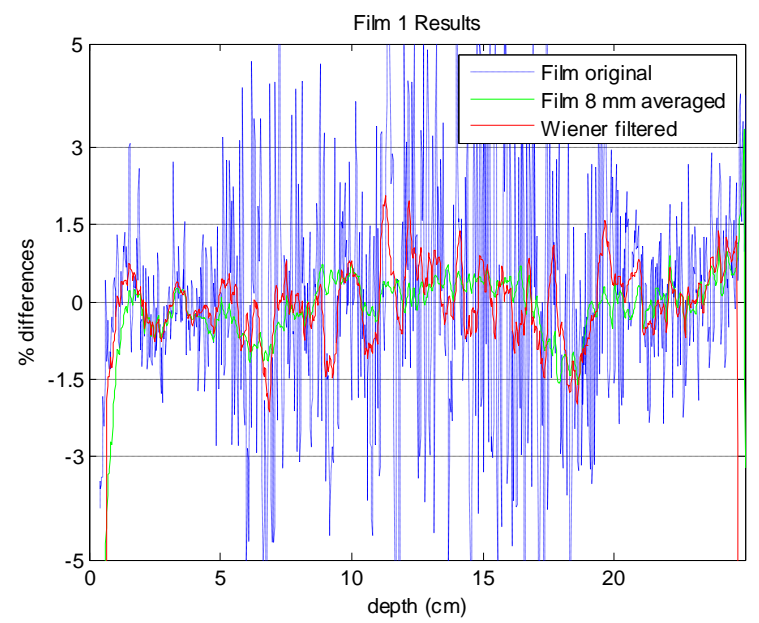

Fig. 4. Thepercentage differences between the normalized delivered dose with the original, averaged and filtered data for the first film

However, according to Fig. 5 and Fig. 6, the percentage difference of Wiener filtered data is obviously better than that of the $8 \times 8 \mathrm{~mm}^{2}$ averaged datato be around $1 \%$. Through Fig. 4 to Fig. 6 , the percentage differences of the original data would be higher than $5 \%$.

\section{CONCLUSION}

A Weiner filter technique was presented and applied in the dosimetry systems of EBT2 film. It was shown to be better or at least equivalent to the traditional averaging out technique, but it offered better spatial resolution than the traditional method. This technique could also be used to perform the dosage quality assurance of treatment planning for the advanced radiation treatment technology, for example, the intensity modulated radiation therapy (IMRT) and volumetric modulated arc therapy (VMAT).

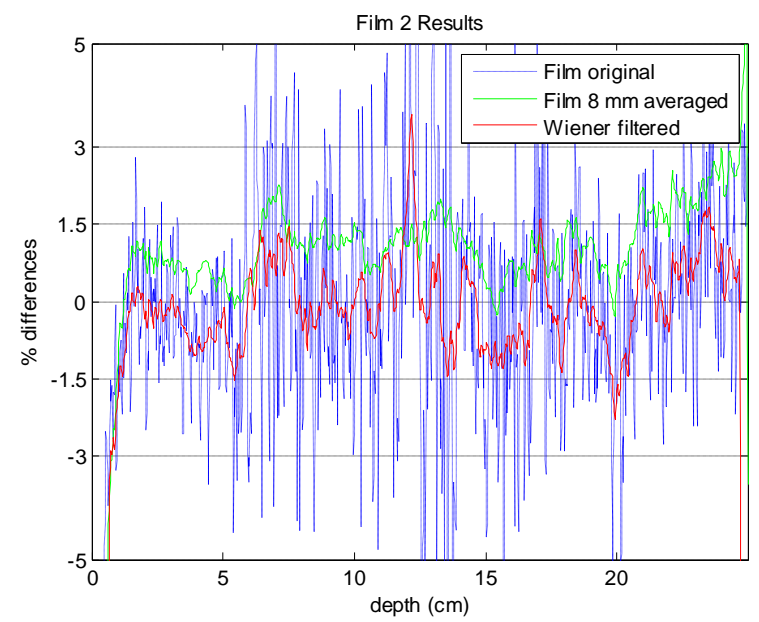

Fig. 5. Thepercentage differences between the normalized delivered dose with the original, averaged and filtered data for the second film

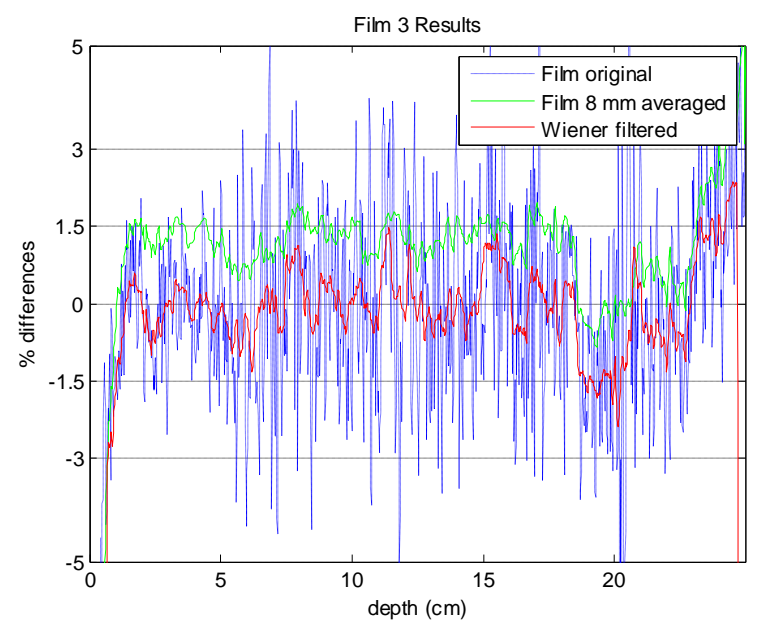

Fig. 6. Thepercentage differences between the normalized delivered dose with the original, averaged and filtered data for the third film.

\section{ACKNOWLEDGMENT}

Research supported by NSC of Taiwan (NSC101-2221-E-214 -019).

\section{REFERENCES}

[1] J. S. Tsai et al., "Dosimetric verification of the dynamic intensity-modulated radiation therapy of 92 patients," Int. J. Radiat Oncol Biol Phys, vol. 40, pp. 1213-1230, Mar. 15, 1998.

[2] D. A. Low et al., "A technique for the quantitative evaluation of dose distributions," Med Phys, vol. 25, pp. 656-61, May 1998.

[3] M. Stock et al., "Interpretation and evaluation of the gamma index and the gamma index angle for the verification of IMRT hybrid plans," Phys Med Biol, vol. 50, pp. 399-411, Feb. 7, 2005.

[4] P. J. Muench et al., "Photon energy dependence of the sensitivity of radiochromic film and comparison with silver halide film and $\mathrm{LiF}$ TLDs used for brachytherapy dosimetry," Med Phys, vol. 18, pp. 769-775, Jul.-Aug. 1991.

[5] A. Palm et al., "Predicting energy response of radiographic film in a 6 MV x-ray beam using Monte Carlo calculated fluence spectra and absorbed dose," Med Phys, vol. 31, pp. 3168-3178, Dec. 2004.

[6] I. J. Yeo et al., "A filtration method for improving film dosimetry in photon radiation therapy," Med Phys, vol. 24, pp. 1943-1953, Dec. 1997.

[7] S. G. Ju et al., "Film dosimetry for intensity modulated radiation therapy: dosimetric evaluation," Med Phys, vol. 29, pp. 351-355, Mar. 2002.

[8] A. N. Rad et al., "Radiochromic film dosimetry: recommendations of AAPM Radiation Therapy Committee Task Group 55. American 
Association of Physicists in Medicine," Med Phys, vol. 25, pp. 2093-2115, Nov. 1998

[9] O. A. G. Garduno et al., "Radiation transmission, leakage and beam penumbra measurements of a micro-multileaf collimator using GafChromic EBT film," J. Appl. Clin Med Phys, vol. 9, pp. 90-98, 2008.

[10] G. Coscia et al., "Fractionated stereotactic radiotherapy: a method to evaluate geometric and dosimetric uncertainties using radiochromic films," Med Phys, vol. 36, pp. 2870-2880, Jul. 2009.

[11] E. E. Wilcox and G. M. Daskalov, "Evaluation of GAFCHROMIC EBT film for Cyberknife dosimetry," Med Phys, vol. 34, pp. 1967-1974, Jun 2007.

[12] A. K. Ho et al., "The use of TLD and Gafchromic film to assure submillimeter accuracy for image-guided radiosurgery," Med Dosim, vol. 33, pp. 36-41, Spring 2008.

[13] M. Fuss et al., "Dosimetric characterization of GafChromic EBT film and its implication on film dosimetry quality assurance," Phys Med Biol, vol. 52, pp. 4211-4225, Jul. 21, 2007.

[14] C. Fiandra et al., "Clinical use of EBT model Gafchromic film in radiotherapy," Med Phys, vol. 33, pp. 4314-9, Nov. 2006.

[15] M. J. Butson et al., "Absorption spectra variations of EBT radiochromic film from radiation exposure," Phys Med Biol, vol. 50, pp. N135-140, Jul. 7, 2005 .

[16] F. Schneider et al., "Optimization of the gafchromic EBT protocol for IMRT QA," Z Med Phys, vol. 19, pp. 29-37, 2009.

[17] L. J. van Battum et al., "Accurate dosimetry with GafChromic EBT film of a $6 \mathrm{MV}$ photon beam in water: what level is achievable?" Med Phys, vol. 35, pp. 704-716, Feb. 2008.

[18] S. Saur and J. Frengen, "GafChromic EBT film dosimetry with flatbed CCD scanner: a novel background correction method and full dose uncertainty analysis," Med Phys, vol. 35, pp. 3094-3101, Jul. 2008

[19] S. Devic et al., "Precise radiochromic film dosimetry using a flat-bed document scanner," Med Phys, vol. 32, pp. 2245-2253, Jul. 2005.

[20] B. Hartmann et al., "Homogeneity of Gafchromic EBT2 film," Med Phys, vol. 37, pp. 1753-1756, Apr. 2010.
[21] B. Arjomandy et al., "Energy dependence and dose response of Gafchromic EBT2 film over a wide range of photon, electron, and proton beam energies," Med Phys, vol. 37, pp. 1942-1947, May 2010

[22] J. G. Sutherland and D. W. Rogers, "Monte Carlo calculated absorbed-dose energy dependence of EBT and EBT2 film," Med Phys, vol. 37, pp. 1110-1116, Mar. 2010.

[23] L. Chang et al., "Calibration of EBT2 film by the PDD method with scanner non-uniformity correction," Phys Med Biol, vol. 57, pp. 5875-5887, 2012.

[24] B. C. Ferreira et al., "Evaluation of an Epson flatbed scanner to read Gafchromic EBT films for radiation dosimetry," Phys Med Biol, vol. 54, pp. 1073-1085, Feb. 21, 2009.

[25] E. E. Klein et al., "Task Group 142 report: quality assurance of medical accelerators," Med Phys, vol. 36, pp. 4197-4212, Sep. 2009.

[26] G. J. Kutcher et al., "Comprehensive QA for radiation oncology: report of AAPM Radiation Therapy Committee Task Group 40," Med Phys, vol. 21, pp. 581-618, Apr. 1994.

[27] R. J. Schulz et al., "A protocol for the determination of absorbed dose from high-energy photon and electron beams," Med Phys, vol. 10, pp. 741-771, Nov.-Dec. 1983.

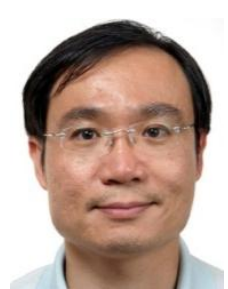

Liyun Chang received the M.S. degree from the Department of radiological physics, Wayne State University, USA, in 1995, and the Ph.D. degree in biomedical engineering from the National Cheng-Kung University, Tainan, Taiwan, in 2009. He is currently an assistant professor in the Department of Medical Imaging and Radiological Sciences, I-Shou University, Kaohsiung, Taiwan. He was a clinical medical physicist in hospital for 15 years. His majorresearch interests include evolutionary optimization and innovative technique applied to the field of radiation therapy. He published more than 10 refereed journal papers and 15 conference papers as first author. 